\title{
LPA2 receptor Agonist Radioprotectin-1 Attenuates Radiation-Induced Apical Junctional Complex Disruption and Barrier Dysfunction in Mouse Colon
}

\author{
Pradeep Shukla ${ }^{1}$, Avtar Meena ${ }^{1}$, Ruchika Gangwar ${ }^{1}$, Erzsebet Szabo ${ }^{1}$, Andrea Balogh ${ }^{1}$, \\ Sue Chin Lee ${ }^{1}$, Alain Vandewalle ${ }^{2}$, Gabor Tigyi ${ }^{1}$, and RadhaKrishna Rao ${ }^{1}$ \\ ${ }^{1}$ University of Tennessee Health Science Center \\ ${ }^{2}$ INSERM U373
}

May 5, 2020

\begin{abstract}
Background and Purpose The colonic epithelium is highly sensitive to ionizing radiation, leading to impaired barrier function. Lysophosphatidic acid (LPA) is protective against radiation-induced intestinal mucosal injury and genotoxic stress. We evaluated the effect of LPA and its analog, Radioprotein-1 in radiation-induced colonic epithelial barrier dysfunction. Experimental approach Caco-2 and m-ICC12 cell monolayers were exposed up to g-radiation, and the barrier function was evaluated by measuring and unidirectional flux of FITC-inulin. Mice were subjected to either total body irradiation (TBI) or partial body irradiation (PBI-BM5). Intestinal barrier function was analyzed by evaluating mucosal permeability to inulin and measuring plasma lipopolysaccharide (LPS) levels. Tight junction and adherens junction integrity was examined by confocal microscopy. Oxidative stress was assessed by measuring protein thiol oxidation and antioxidant mRNA. Key Results In Caco-2 and m-ICC12 cell monolayers, LPA attenuates radiation-induced redistribution of tight junction proteins from the junctions, which was blocked by Rho-kinase inhibitor. In mice, TBI and PBI-BM5 disrupt colonic epithelial tight junction and adherens junction, increases mucosal inulin permeability and elevates plasma LPS. RP1 administered 30 min pre-irradiation or 24 hours post-irradiation alleviates TBI and PBI-BM5-induced tight junction disruption, barrier dysfunction, and endotoxemia. The RP1 effects on radiation-induced colonic injury was associated with protein thiol oxidation, suppression of antioxidant gene expression, cofilin activation and remodeling of actin cytoskeleton. Conclusion and Implications These data demonstrate that LPA2 receptor agonists prevent and mitigate g-irradiation-induced colonic mucosal barrier dysfunction and endotoxemia, indicating their potential therapeutic benefit in the treatment of the gastrointestinal acute radiation syndrome.
\end{abstract}

\section{Introduction}

Radiological accidents or belligerent use of a nuclear device is an ever-present global concern. Thus, the development of radiation countermeasures that mitigate the acute radiation syndrome (ARS) is a priority for every nation to protect first responders, the military, and civilians at large. At this time, no FDA approved radiomitigator exists. The gastrointestinal (GI) tract has been considered as a highly vulnerable target of irradiation. The radiation-induced GI dysfunction is commonly referred to as GI-ARS. GI-ARS is characterized by rapid-onset diarrhea during the early stage of radiation injury, followed by endotoxemia and bacteremia, leading to septicemia in the later stage. The fact that endotoxemia and bacteremia are essential events in the pathogenesis of ARS and the colonic bacteria are the primary source of endotoxins highlights the critical importance of colonic tissue injury in the pathogenesis of ARS. Endotoxemia and bacteremia are significant factors involved in multiple organ dysfunction in ARS.

Typically, the colonic epithelial tight junction and mucosal barrier function prevent diffusion of endotoxins and bacteria from the lumen into the colonic tissue (Anderson \& Van Itallie, 2009; Rao, 2008; Van Itallie 
\& Anderson, 2006). The tight junction, a multiprotein complex that seals the intercellular space of the epithelium, is tightly regulated by cell signaling networks (Basuroy, Seth, Elias, Naren \& Rao, 2006; Basuroy, Sheth, Kuppuswamy, Balasubramanian, Ray \& Rao, 2003; Rao, 2008; Rao, Basuroy, Rao, Karnaky Jr \& Gupta, 2002; Seth, Sheth, Elias \& Rao, 2007; Sheth, Samak, Shull, Seth \& Rao, 2009b; Sheth et al., 2007). Occludin, one of the transmembrane protein component of the tight junction, interacts with a scaffold protein ZO-1, which is an essential interaction for the assembly and maintenance of tight junction. Adherens junction is located beneath the tight junction and is known to indirectly regulate the integrity of tight junction (Rao, Seth \& Sheth, 2004). The tight junction and adherens junction integrated to the underlying actin cytoskeleton define the apical junctional complex (AJC) of the epithelium. Our recent study indicated that the tight junction and adherens junction of the mouse colon are highly sensitive to radiation (Shukla et al., 2016). These junctions are disrupted as early as two hours post-irradiation with sustained damage lasting at least for five days. These findings emphasize the importance of early disruption of colonic epithelial tight junction and barrier dysfunction in leading to endotoxemia, bacteremia, and septicemia in GI-ARS. Understanding the cellular and molecular mechanisms of this ARS that we propose to designate as "Colonic Radiation Sub-syndrome" is crucially important for the development of effective radiomitigators and treatment strategies.

Lysophosphatidic acid (LPA) is a growth factor-like lipid mediator that rescues cells from genotoxic stress, including radiation-induced apoptosis and cellular injury in vitro and in vivo when administered postirradiation (Deng, Balazs, Wang, Van Middlesworth, Tigyi \& Johnson, 2002). Exogenously delivered LPA, due to its short half-life in plasma $(\sim 9 \mathrm{~min})$, is not suitable for use as a therapeutic. However, stabilized analogs of LPA such as Radioprotectin-1 [RP1, (Patil et al., 2014; Patil et al., 2015)] have radioprotective (Deng, Balazs, Wang, Van Middlesworth, Tigyi \& Johnson, 2002; Deng et al., 2015; Deng et al., 2007) as well as radio-mitigative (Deng et al., 2015; Kiss et al., 2013) efficacy in animal models of GI-ARS. In vitro receptor add-back studies and experiments conducted with Lpar2 knockout (Lpar - $^{-/-}$) mice indicate that this receptor subtype is necessary and sufficient to protect cells and mice from radiation-induced apoptotic cell death and tissue injury to the gut (Deng et al., 2007; Lin, Lai, Makarova, Tigyi \& Lin, 2007). Thus, improved non-lipid analogs specific to the LPA2 receptor such as RP1could be suitable for the treatment of colonic ARS without activation of the other LPA receptor subtypes.

In this study, we evaluated the protective and mitigating effects of RP1 on the radiation-induced disruption of intestinal epithelial AJC, an increase in mucosal permeability, and endotoxemia.

\section{Methods}

\section{Cell culture}

Caco-2 $2_{\text {bbe2 }}$ (ATCC, Rockville, MD) and m-IC $\mathrm{Cl}_{2}$ (a kind gift from Dr. Alain Vandewalle, INSERM U773, Paris, France; (Bens et al., 1996)) cells were grown under standard cell culture conditions as previously described (Sheth, Samak, Shull, Seth \& Rao, 2009a). Experiments were conducted using cells grown in Transwell inserts of varying diameters $(6.5$ or $12 \mathrm{~mm}$ ) for $3-4$ or $15-17$ days.

Epithelial barrier function

Transepithelial macromolecular permeability was evaluated by measuring the unidirectional flux of FITCinulin as previously described (Sheth, Samak, Shull, Seth \& Rao, 2009a).

Animals

All animal studies were performed under the protocols approved by the University of Tennessee Health Science Center (UTHSC) Institutional Animal Care and Use Committee (IACUC). In the first study, 12-14 week-old adult wild type (WT) and Lpare $^{-/-}$mice were subjected to total body irradiation (TBI; 9.5 Gy). WT and $L$ par2 $^{-/-}$mice were randomized into Sham (sham-treated), and IR (irradiated) groups. The integrity of colonic epithelial tight junction and adherens junction were examined at 2 hours after TBI. In the first study, mice were subjected to TBI with or without RP-1 administration $(0.1 \mathrm{mg} / \mathrm{kg}$, i.p., single dose 24 hours before irradiation, pretreatment group). Control mice were subjected to similar conditions without 
radiation (sham-treated). In the second study, mice were randomized into three groups: Sham (control), IR (irradiated with the administration of vehicle), and IR+ RP-1 (irradiated with the administration of RP1 at 24 hours post-irradiation). The integrity of colonic epithelial tight junction, adherens junction, and endotoxemia was examined at 2 and 4 hours post-irradiation. In the third study, mice were subjected to partial body irradiation, in which $5 \%$ of the bone marrow was shielded (PBI-BM5). Experimental groups consisted of Sham (control), IR (irradiated with the administration of vehicle), and IR+RP-1 (irradiated with intraperitoneal administration of RP1; $3 \mathrm{mg} / \mathrm{kg}$, s.c.; at 24 hours post-irradiation). At varying times (28, 52,76 , and100 hours) after irradiation, the integrity of colonic epithelial tight junction, adherens junction, and actin cytoskeleton was examined. At 28 hours post-irradiation, colons were collected and analyzed for oxidative stress and epithelial junctional integrity.

Irradiation

Cells were irradiated with 2, 5, or 10 Gy $\gamma$-irradiation from a ${ }^{137} \mathrm{Cs}$ source (using a J.L. Shepherd \& Assoc. Mark I, Model 25, San Fernando, CA, USA) at a dose rate of $440 \mathrm{cGy} / \mathrm{min}$. After irradiation, the culture medium was replaced with fresh complete culture medium. For TBI, mice were subjected to radiation $(9.5$ Gy at a dose rate of $76 \mathrm{cGy} / \mathrm{min})$. For the PBI model, mice were anesthetized with $87 \mathrm{mg} / \mathrm{kg}$ ketamine and $13 \mathrm{mg} / \mathrm{kg}$ xylazine IP and placed in a plexiglass restrainer so that the legs below the knee were shielded. The shielding protected tibiae, fibulae, and the paws from radiation that contained $5 \%$ of the bone marrow. Mice placed into the isodose field of the irradiator were irradiated in groups of eight. In this model, 15.7 Gy delivered at a dose rate of $147 \mathrm{cGy} /$ min yielded empirical $\mathrm{LD}_{85 / 10}$ mortality. Radiation field mapping and calibration by ion chamber dosimetry were done by the manufacturer. In addition, routine validation and quality control measurements of exposure rates and exposure rate mapping in the chamber at positions of interest were conducted by a certified health physicist using a calibrated RadCal 0.6 cc therapy grade ion chamber/electrometer system. High-dose thermoluminescent dosimeters were used in most irradiations to validate the actual dose delivered to the mice (calibrated at MD Anderson Cancer Center Radiation Dosimetry Services). The isodose field was validated using Gafchromic film for high-dose dosimetry (10-50 Gy, Ashland Inc., Covington, KY). At the end of the experiment, gut permeability was measured as described below. Colon segments were stored frozen for further analyses.

Gut permeability in vivo

Mucosal barrier dysfunction in vivo was evaluated by measuring gut permeability to FITC-inulin (6 kDa). On the last day of the experiment, mice were injected with FITC-inulin $(50 \mathrm{mg} / \mathrm{ml}$ solution; $2 \mu \mathrm{l} / \mathrm{g}$ body weight) via the tail vein. One hour after injection, blood samples were collected by cardiac puncture under isoflurane anesthesia, and plasma was isolated using heparin sulfate anticoagulant. Luminal contents from the colon and ileum were flushed with $0.9 \%$ saline. Fluorescence in plasma and the luminal flushing was measured using a fluorescence plate reader. Fluorescence values in the luminal flushing were normalized to fluorescence values in corresponding plasma samples and calculated as the percent of the amount injected.

Immunofluorescence microscopy

Caco-2 and $\mathrm{m}-\mathrm{IC}_{\mathrm{C} 12}$ cell monolayers were permeabilized with $0.2 \%$ Triton $\mathrm{X}-100$, blocked, and stained for different junctional proteins as described in a prior study (Shukla et al., 2018). Fluorescence was examined by using a Zeiss LSM 510/710 laser scanning confocal microscope. Cryo-sections of the colon (10 $\mu \mathrm{m}$ thickness) were fixed in acetone and methanol mixture $(1: 1)$ at $20^{\circ} \mathrm{C}$ for two minutes and rehydrated in 14 $\mathrm{mM}$ phosphate-buffered saline (PBS). Sections were permeabilized with $0.5 \%$ Triton X-100 in PBS for 15 minutes and blocked in $4 \%$ non-fat milk in TBST (20 mM Tris, $\mathrm{pH} 7.2$ and $150 \mathrm{mM} \mathrm{NaCl}$ ). Sections were incubated for one hour with primary antibodies (mouse monoclonal anti-occludin and rabbit polyclonal antiZO-1 antibodies or mouse monoclonal E-cadherin and rabbit polyclonal anti- $\beta$-catenin antibodies), followed by incubation for one hour with the secondary antibodies (AlexaFluor-488-conjugated anti-mouse IgG and Cy3-conjugated anti-rabbit IgG antibodies from Molecular Probes, Eugene, OR) containing Hoechst 33342 dye. In all cases, images from x-y $(1 \mu \mathrm{m})$ sections were collected using LSM Pascal or Zen software (White Plains, NY, USA). Images from optical sections were stacked using ImageJ software (NIH, Bethesda, MD, 
USA) and processed with Adobe Photoshop (Adobe Systems, San Jose, CA, USA).

Preparation of the detergent-insoluble fraction

Actin-rich detergent-insoluble fraction was prepared as described previously (Rao, Basuroy, Rao, Karnaky \& Gupta, 2002). Mucosal scrapping from the colon and ileum were incubated on ice for 15 minutes with lysis buffer-CS (Tris buffer containing 1\% Triton-X100, $2 \mu \mathrm{g} / \mathrm{ml}$ leupeptin, $10 \mu \mathrm{g} / \mathrm{ml}$ aprotinin, $10 \mu \mathrm{g} / \mathrm{ml}$ bestatin, $10 \mu \mathrm{g} / \mathrm{ml}$ pepstatin- $\mathrm{A}, 10 \mu \mathrm{l} / \mathrm{ml}$ of protease inhibitor cocktail, $1 \mathrm{mM}$ sodium vanadate and $1 \mathrm{mM}$ PMSF). Briefly, mucosal lysates were centrifuged at $15,600 \mathrm{x}$ g for $4 \mathrm{~min}$ at $4^{\circ} \mathrm{C}$ to sediment the high-density actin-rich detergent-insoluble fraction. The pellet was suspended in $100 \mu$ of preheated lysis buffer-D $(20 \mathrm{mM}$ Tris buffer, $\mathrm{pH} 7.2$, containing $10 \mu \mathrm{l} / \mathrm{ml}$ of protease inhibitor cocktail, $10 \mathrm{mM}$ sodium fluoride, $1 \mathrm{mM}$ sodium vanadate and $1 \mathrm{mM} \mathrm{PMSF}$ ), sonicated to homogenize the actin cytoskeleton, and heated at $100^{\circ} \mathrm{C}$ for $10 \mathrm{~min}$. Protein content was measured by the BCA method (Pierce Biotechnology, Rockford, IL). Triton-insoluble and soluble fractions were mixed with an equal volume of $2 \mathrm{X}$ concentrated Laemmli's sample buffer, heated at $100^{\circ} \mathrm{C}$ for $5 \mathrm{~min}$, and $25-40 \mu \mathrm{g}$ protein samples were used for SDS-polyacrylamide gel electrophoresis followed by immunoblot analysis.

Immunoblot analysis

Triton-soluble and insoluble fractions were separated by SDS-polyacrylamide gel electrophoresis (7\%) and transferred to PVDF membranes as described in a previous study (Rao, Basuroy, Rao, Karnaky \& Gupta, 2002). Membranes were immunoblotted for different proteins using specific antibodies for different tight junction and adherens junction proteins with $\beta$-actin as the housekeeping protein in combination with HRPconjugated anti-mouse IgG or anti-rabbit IgG secondary antibodies. Blots were developed using the ECL chemiluminescence reagent (Pierce, Rockford, IL) and quantitated by densitometry using ImageJ software. The density for each band was normalized to the density of the corresponding actin band.

Protein thiol assay

Protein thiols in colonic sections were monitored as described previously (Shukla et al., 2018). Briefly, reduced protein thiols were evaluated by staining cryosections of the colon with BODIPY FL- $N$-(2-aminoethyl) maleimide (FLM) and confocal microscopy at excitation and emission wavelengths (490 nm and $534 \mathrm{~nm}$, respectively). For oxidized protein thiols, the reduced protein thiol was first alkylated with N-ethylmaleimide followed by reduction of oxidized protein thiols with tris (2-carboxyethyl) phosphine prior to staining with FLM. Control staining was done after N-ethylmaleimide treatment. Fluorescence images were collected and quantitated using ImageJ software.

RNA extraction and RT-qPCR

RNA was isolated from the colon by using the TRIzol kit (Invitrogen, Carlsbad, CA, USA) and quantified using a NanoDrop photometer. Total RNA $(1.5 \mu \mathrm{g})$ was used for the generation of cDNA using the ThermoScript RT-PCR kit for first-strand synthesis (Invitrogen). Quantitative PCR (qPCR) reactions were performed using cDNA mix (cDNA corresponding to $35 \mathrm{ng}$ RNA) with 300 nmole primers in a final volume of $25 \mu \mathrm{l}$ of $2 \times$ concentrated RT2 Real-Time SYBR Green/ROX master mix (Qiagen, Germantown, MD, USA) in an Applied Biosystems QuantStudio 6 Flex Real-Time PCR instrument (Norwalk, CT, USA). The cycle parameters were: $50 \mathrm{degC}$ for $2 \mathrm{~min}$, one denaturation step at $95 \mathrm{degC}$ for $10 \mathrm{~min}$, and 40 cycles of denaturation at $95 \operatorname{deg} \mathrm{C}$ for $10 \mathrm{~s}$, followed by annealing and elongation at $60 \mathrm{deg}$. The relative gene expression of each transcript was normalized to the GAPDH gene transcripts using the $\Delta \Delta \mathrm{Ct}$ method. Sequences of primers used for qPCR are provided in the Supplemental Information section (Table-1).

Plasma endotoxin assay

Plasma endotoxin concentrations were measured using Pierce LAL Chromogenic Endotoxin Quantitation Kit (Thermo Scientific, Cat\# 88282) according to the manufacturer's instructions.

Statistical Analysis 
All data are expressed as Mean \pm SEM. The differences among multiple groups were first analyzed by ANOVA (Prism 6.0, GraphPad, Inc. San Diego, CA, USA). When statistical significance was detected, Tukey's t-test was used to determine the statistical significance between multiple testing groups and the corresponding control. Statistical significance was established at $95 \%$ confidence.

Materials

Hoechst 33342 dye and BODIPY FL- $N$-(2-aminoethyl) maleimide were purchased from Life Technologies (Grand Island, NY, USA). N-ethylmaleimide and tris (2-carboxyethyl) phosphine were from Sigma-Aldrich (St Louis, MO, USA). All other chemicals were purchased from either Sigma-Aldrich or Thermo Fisher Scientific (Tustin, CA, USA). Anti-ZO-1, anti-occludin, anti-claudin-2 (Cldn-2) and anti-claudin-3 (Cldn3) antibodies were purchased from Invitrogen (Carlsbad, CA, USA). Anti-E-Cadherin and anti- $\beta$-catenin antibodies were purchased from BD Biosciences (Billerica, MA, USA). Horseradish peroxidase-conjugated anti-mouse IgG and anti-rabbit IgG and anti- $\beta$-actin antibodies were obtained from Sigma-Aldrich (St Louis, MO, USA). AlexaFlour-488-conjugated anti-mouse IgG and Cy3-conjugated anti-rabbit IgG were purchased from Molecular Probes (Eugene, OR, USA). The anti-nrf2 antibody was purchased from Abcam (Cambridge,

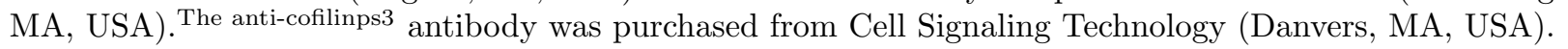

\section{Results}

LPA attenuates radiation-induced disruption of tight junctions and blocks barrier dysfunction in the intestinal epithelium

Caco-2 (Fig. 1, A-D) and m- $\mathrm{IC}_{\mathrm{C} 12}$ (Fig. 1, E) cell monolayers were incubated with LPA $(10 \mu \mathrm{M}) 30 \mathrm{~min}$ before irradiation at varying doses, and the tight junction integrity was examined by confocal microscopy for occludin and ZO-1. In control Caco-2 cell monolayers, occludin, and ZO-1 were co-localized at the intercellular junctions indicating the presence of intact tight junctions (Fig. 1A). Irradiation induced a dose-dependent redistribution of tight junction proteins, whereas pretreatment of the epithelium with LPA attenuated radiation-induced redistribution of tight junction proteins at both 5 Gy and 10 Gy doses. Densitometric analysis of ZO-1 fluorescence at the intercellular junctions indicated a significant reduction in junctional localization of ZO-1 post-irradiation. LPA-treated samples showed attenuation of radiation-induced ZO-1 redistribution (Fig. 1B). Tight junction protein complexes are anchored to the actin cytoskeleton. The disruption of the actin cytoskeleton is known to disrupt tight junction (Madara, Stafford, Barenberg \& Carlson, 1988). Cofilin is an actin severing protein that plays an essential role in the disruption of the actin cytoskeleton. Fluorescence staining for $\mathrm{F}$-actin and cofilin ${ }^{\mathrm{pS} 3}$ (the inactive form) showed that radiation causes disruption of F-actin filaments and reduces the levels of cofilin ${ }^{\mathrm{pS} 3}$. Pretreatment with LPA blocked radiation-induced reduction of inactive cofilin and disruption of F-actin filaments (Fig. 1C). Rho-GTPase is known to be involved in the activation of cofilin and the promotion of actin cytoskeletal organization (Vardouli, Moustakas \& Stournaras, 2005; Wang, Halasz \& Townes-Anderson, 2019). The data showed that toxin-B, an inhibitor of Rho-GTPase, blocked LPA-mediated prevention of radiation effects on cofilin and F-actin organization (Fig. 1D). To confirm the effects of radiation on the tight junction, we examined the effects of radiation and LPA in I- $\mathrm{mC}_{\mathrm{C} 12}$ cell monolayers, a mouse intestinal epithelial cell line (Bens et al., 1996). Data showed that radiation-induced a dose-dependent redistribution of ZO-1 from the intercellular junctions, and LPA pretreatment attenuated this effect of radiation (Fig. 1E).

Effect of $\mathrm{LPA}_{2}$ deficiency on the radiation-induced disruption of tight junction and adherens junction in the mouse colon

in vivo

The $\mathrm{LPA}_{2}$ receptor plays a crucial role in many intestinal mucosal protective functions of LPA, including inhibition of cholera toxin-induced diarrhea (Li et al., 2005), intestinal ion transport (Lin et al., 2010; Singla et al., 2010; Thompson et al., 2018), prevention of radiation-induced intestinal mucosal atrophy and stem cell ablation (Kuo et al., 2018). In this study, we examined the effect of $\mathrm{LPA}_{2}$ deficiency on the severity of radiation-induced disruption of colonic epithelial tight junctionsin vivo at 2 hours after 
irradiation. Immunofluorescence confocal images show that redistribution of occludin and ZO-1 from the epithelial junctions was more severe in Lpar2 ${ }^{-/}$mice compared to that present in WT mouse colons (Fig. 2A). Similarly, radiation-induced redistribution of adherens junction proteins, E-cadherin, and $\beta$-catenin from the epithelial junctions was more severe in the colon of Lpar2 ${ }^{-/-}$mice, compared to that of WT mice (Fig. 2B).

RP1 blocks radiation-induced disruption of apical junctional complexes, barrier dysfunction, and endotoxemia

RP1, a stable non-lipid $\mathrm{LPA}_{2}$-specific agonist (Patil et al., 2014), protects the intestinal mucosa from diarrhea and mucosal atrophy by various types of insults (Kuo et al., 2018; Patil et al., 2015; Thompson et al., 2018). In the current study, we evaluated the effect of RP1 on the radiation-induced disruption of intestinal epithelial tight junctions and adherens junctions, the increase in mucosal permeability, and endotoxemia. Prophylactic administration of RP1 (0.5 mg/kg; s.c.) at $30 \mathrm{~min}$ pre-irradiation blocked the radiation-induced loss of junctional distribution of occludin and ZO-1 when examined at 2 hours post-irradiation (Fig. 3A). Similarly, RP1 administration blocked radiation-induced redistribution of E-cadherin and $\beta$-catenin from the epithelial junctions (Fig. 3B). Densitometric analysis of ZO-1 (Fig. 3C) and E-cadherin (Fig. 3D) fluorescence at the junctions indicated that RP1 completely attenuated radiation-induced redistribution of these proteins from the junctions. Mucosal permeability in the colon and ileum in vivo was measured at 2 hours and 4 hours after irradiation. RP1 significantly reduced radiation-induced mucosal permeability in the colon (Fig. 3E). RP1 showed no significant effect in the ileum at these time points (Fig. 3F). Prevention of radiation-induced colonic mucosal permeability by RP1 at 4 hours post-irradiation was associated with a significant $(\mathrm{p}=0.04)$ reduction of the radiation-induced increase in plasma LPS levels (Fig. 3G).

RP1 mitigates radiation-induced disruption of apical junctional complexes, barrier dysfunction, and endotoxemia

In this study, RP1 was administered 3 hours after irradiation $(0.5 \mathrm{mg} / \mathrm{kg}$ daily; s.c.). The post-irradiation treatment with RP1 reversed the radiation-induced loss of junctional occludin and ZO-1 when examined at 24 hours post-irradiation (Fig. 4A). Similarly, RP1 reversed radiation-induced redistribution of E-cadherin and $\beta$-catenin from the epithelial junctions (Fig. 4B). Radiation also induced redistribution of claudin-3, another transmembrane protein of tight junction in mouse colons (Fig. 4C). Post-irradiation treatment with RP1 reversed this effect of radiation on the colonic epithelial distribution of claudin-3. Densitometric analysis of ZO-1 (Fig. 4D), $\beta$-catenin (Fig. 4E), and claudin-3 (Fig. 4F) at the junctions confirmed that RP1 completely attenuated radiation-induced redistribution of these proteins from the junctions. Mucosal permeability in colon and ileum in vivo was measured at 24 and 48 hours post-irradiation. RP1 significantly alleviated radiation-induced mucosal permeability in the colon (Fig. 4G) and ileum (4H). Restoration of radiation-induced loss of intestinal mucosal permeability by RP1 was associated with a significant $(\mathrm{p}=$ 0.015 at 24 hours \& 0.0001 at 48 hours) reduction of radiation-induced elevation of plasma LPS (Fig. 4I).

RP1 mitigates radiation-induced suppression of antioxidant gene expression

Our previous study demonstrated that radiation induces oxidative stress and the antioxidant N-acetyl-Lcysteine blocks as well as mitigates radiation-induced disruption of tight junctions and barrier dysfunction in mouse colons (Shukla et al., 2016). In this study, we evaluated the effect of RP1 on radiation-induced effects on antioxidant gene expression. RP1 (0.5 mg/ $\mathrm{kg}$ daily; s.c.), administered at 24 hours post-irradiation, reversed the radiation-induced reduction of mRNA forGpx1 (Fig. 5A), Sod1 (Fig. 5B), and Prdx1 (Fig. $5 \mathrm{C}$ ). However, RP1 failed to reverse radiation-induced reduction of $C A T$ (catalase) mRNA (Fig. 5D). The mRNA levels for Nrf2, the transcription factor involved in antioxidant gene expression, was reduced by radiation; however, it was reversed by $\mathrm{RP} 1$ treatment (Fig. 5E).

Effects of RP1 on tight junction and adherens junction integrity in the PBI-BM5 model

The impact of PBI-BM5 on the intestinal epithelial tight junction integrity and its prevention by RP1 treatment were evaluated next. Immunofluorescence confocal microscopy showed that PBI-BM5 induced a 
redistribution of occludin and ZO-1 from the colonic epithelial junctions when examined at 52 hours postirradiation, and RP1 (3 mg/kg daily; s.c.; beginning 24 hours after irradiation) treatment alleviated this effect of RP1 (Fig. 6A). Similarly, PBI induced redistribution of adherens junction proteins, E-cadherin, and $\beta$-catenin from the epithelial junctions and RP1 alleviated this effect of PBI (Fig. 6B). Densitometric analysis for ZO-1 fluorescence at the junctions indicated that PBI-BM5 did not affect tight junction integrity at 28 hours post-irradiation but induced a severe disruption of tight junctions at 52 and 76 hours (Fig. 6C). RP1 treatment completely blocked PBI-induced ZO-1 redistribution at both 52 and 76 hours post-irradiation. RP1 also prevented PBI-induced reduction of junctional E-cadherin levels (Fig. 6D).

RP1 attenuates PBI-BM5-induced oxidative stress in colonic mucosa

Protein thiol oxidation was examined by fluorescence staining of reduced-protein thiols and oxidized-protein thiols at 52 hours post-irradiation. Fluorescence images (Fig. 7A) and densitometric analyses (Fig. 7B) indicated that PBI-BM5 depleted reduced protein thiols with a corresponding increase in oxidized protein thiols and that RP1 treatment (3 mg/kg daily; s.c.; beginning 24 hours after irradiation) reversed PBIinduced protein thiol oxidation. The expression of Nrf2 was examined by immunofluorescence staining (Fig. 7C), immunoblot analysis (Fig. 7D), and Nrf2 -specific mRNA measurement (Fig. 7E). All these analyses indicated that PBI significantly reduced $N r f 2$ expression, while RP1 blocked this effect of PBI. PBI significantly reduced the levels of mRNA forSOD1 (Fig. 7F), Gpx1 (Fig. 7G), CAT (catalase) (Fig. 7H), Trx1 (Fig. 7I), and Prdx1 (Fig. 7J), and RP1 attenuated these effects of PBI.

RP1 attenuates PBI-BM5-induced F-actin remodeling and its association of apical junctional complexes

The association of tight junction and adherens junction proteins with the actin cytoskeleton was assessed by Western blot analysis of the actin-rich, detergent-insoluble fraction for tight junction and adherens junction proteins. Densitometric analysis of immunoblot bands (Fig. 8A) for E-cadherin (Fig. 8B), $\beta$-catenin (Fig. 8C), Claudin-3 (Fig. 8D), and ZO-1 (Fig. 8E) indicated that PBI-BM5 significantly reduced the detergentinsoluble fraction of E-cadherin, $\beta$-catenin, and claudin-3, which was blocked by RP-1 treatment $(3 \mathrm{mg} / \mathrm{kg}$ daily; s.c.; beginning 24 hours after irradiation). Although PBI-BM5 did not reduce ZO-1 levels, nevertheless RP1 significantly increased ZO-1 density. Immunofluorescence analysis of F-actin showed that PBI reduced F-actin levels, and RP1 treatment attenuated this effect of PBI-BM5 (Fig. 8F). Immunofluorescence staining (Fig. 8G) and immunoblot analysis (Fig. 8H) indicated that PBI-BM5 reduced the levels of cofilin ${ }^{\mathrm{pS} 3}$ in the colonic mucosa, which was blocked by RP1 treatment.

\section{Discussion}

A significant body of evidence indicates that LPA plays a crucial role in the growth and differentiation of intestine as well as the protection of intestinal mucosa from a variety of noxious conditions (Konno et al., 2019; Li et al., 2005; Lin et al., 2010; Thompson et al., 2018; Yun \& Kumar, 2015). However, the role of LPA in the mechanism of protection of the colonic mucosal barrier function is poorly understood. In this new study, we provide evidence that $\mathrm{LPA}_{2}$ receptor activation is radioprotective in the colon by demonstrating that the $\mathrm{LPA}_{2}$ receptor agonist RP1 protects the intestinal epithelium against radiation-induced disruption of apical junctional complexes and prevent or alleviate barrier dysfunction.

The results of in vitro studies in transformed Caco-2 and non-transformed $\mathrm{m}-\mathrm{IC}_{\mathrm{C} 12}$ epithelial monolayers showed that radiation induces a rapid disruption of intestinal epithelial tight junctions in a dose-dependent manner (Fig.1B). In our previous study, we have reported that radiation causes disruption of mouse colonic epithelial tight junctions in vivo as early as 2 hours post-irradiation (Shukla et al., 2016). The present in vitro data demonstrate that radiation directly affects the intestinal epithelium without the systemic influences. The protective effect of $\mathrm{LPA}_{2}$ in irradiated Caco-2 and m-IC $\mathrm{Cl}_{2}$ cell monolayers indicate that LPA directly interacts with the epithelial cells to attenuate radiation-induced tight junction disruption. Irradiation of Caco-2 cell monolayers was associated with a reduction in the inactive form levels of cofilin ${ }^{\mathrm{pS} 3}$. This decrease in cofilin ${ }^{\mathrm{pS} 3}$ levels without change in total cofilin indicates that radiation causes cofilin activation. Disruption of the actin cytoskeleton was previously shown to disrupt tight junctions, which is a common mechanism involved in tight junction disruption mediated by various injurious factors (Madara, Moore \& Carlson, 
1987). Therefore, activation of cofilin, an actin severing protein, is likely involved in radiation-induced tight junction disruption and disruption of the actin cytoskeleton. Cofilin is known to be inactivated by LIM kinase-mediated phosphorylation on S3 residue (Chen \& Macara, 2006), and LPA activates the RhoROCK-LIM kinase pathway (Pandey, Goyal \& Siess, 2007). Abrogation of LPA-mediated protection of tight junctions by the Rho-GTPase inhibitor Toxin-B suggests that LPA likely promotes cofilin phosphorylation by Rho-Rock-LIM kinase pathway.

Previous studies indicated that the intestinal mucosal protective effects of LPA are mediated by activation of $\mathrm{LPA}_{2}$ receptor (Deng, Balazs, Wang, Van Middlesworth, Tigyi \& Johnson, 2002; Deng et al., 2015; Deng et al., 2007; Elias et al., 2009; Khurana et al., 2008; Kuo et al., 2018; Li et al., 2005; Lin, Lai, Makarova, Tigyi \& Lin, 2007; Thompson et al., 2018; Yoshida, He \& Yun, 2016). Our data in the present study show that radiation-induced disruption of tight junction and adherens junction is more severe in $\mathrm{LPA}_{2}$ deficient mice compared to that in WT mice, suggesting that $\mathrm{LPA}_{2}$ activity exerts a protective effect on the intestinal epithelial tight junctions and adherens junctions in vivo. The role of $\mathrm{LPA}_{2}$ receptor in the protection of tight junction was further determined by evaluating the effect of an $\mathrm{LPA}_{2}$-selective agonist, RP1 (Patil et al., 2014), on the radiation-induced disruption of intestinal junctions. The prevention of radiation-induced redistribution of tight junction and adherens junction proteins from the intercellular junctions by RP1pretreatment indicates that $\mathrm{RP} 1$ and $\mathrm{LPA}_{2}$ receptor activation blocks radiation-induced disruption of AJC in the mouse colon. Radiation-induced disruption of tight junction was associated with an increase in mucosal permeability to inulin, indicating the radiation-induced barrier disruption in the mouse colon in vivo . Furthermore, barrier dysfunction was associated with an increase in the levels of plasma LPS in irradiated mice, demonstrating radiation-induced endotoxemia. Prophylactic administration of RP1 blocked radiation-induced colonic mucosal permeability and endotoxemia. Therefore, data from this part of the study indicates that $\mathrm{LPA}_{2}$ activation via prophylactic RP1-treatment prevents radiation-induced disruption of intestinal epithelial AJC, mucosal barrier dysfunction, and endotoxemia.

In order to determine whether RP1 can mitigate radiation-induced intestinal barrier dysfunction, we evaluated the effect of RP1 when administered at 24 hours post-irradiation in mice in vivo . Results of this study showed that RP1 completely reversed radiation-induced redistribution of occludin, ZO-1, claudin-3, E-cadherin and $\beta$-catenin, indicating the reversibility of radiation-induced disruption of tight junctions and adherens junctions and the presence of functioning $\mathrm{LPA}_{2}$ in the irradiated enterocytes. RP1 also reversed the radiation-induced increase in ileal and colonic mucosal permeability to inulin and endotoxemia. Data demonstrates that the $\mathrm{LPA}_{2}$ is an effective target for the treatment of radiation injury to the gut, and RP1 is effective in alleviating radiation-induced intestinal damage. Our previous study indicated that radiation caused oxidative stress in the colonic mucosa and that N-acetylcysteine, the antioxidant, effectively blocked radiation-induced disruption of colonic epithelial tight junctions and barrier dysfunction (Shukla et al., 2016). In the present study, we examined the effect of RP1 on radiation-induced oxidative stress by measuring the levels of antioxidant genes in the colonic mucosa. Significant reduction of mRNA for Gpx1, SOD1 ,Prdx1, $C A T$ (catalase), and Nrf2 indicated that radiation suppresses antioxidant gene expression in colonic mucosa. Data also showed that RP1 completely reversed radiation-induced suppression of Gpx1, SOD1, and Prdx1 expression, and partially reversed the effect of radiation on Nrf2 expression; RP1 showed no significant impact on catalase expression. Therefore RP1-mediated activation of the $\mathrm{LPA}_{2}$ receptor provides significant protection against oxidative damage by irradiation.

All of the above studies applied the TBI model to evaluate the effects of RP1. To mimic the conditions of the GI-ARS, we assessed the effect of RP1 in the PBI-BM5 model in which the shielded bone marrow expands and allows for the survival of the animal proviso protection of the gut mucosa. At 28 hours postirradiation, the junctional distribution of tight junction and adherens junction proteins were unaffected, indicating that PBI-BM5 did not affect intestinal tight junctions and adherens junctions until 28 hours post-irradiation. This PBI effect is in contrast to TBI effects, which disrupted tight junctions within 2 hours post-irradiation. However, by 52 hours post-irradiation, PBI-BM5 caused a severe loss in the junctional distribution of occludin, ZO-1, E-cadherin, and $\beta$-catenin, which was sustained at least until 76 hours. RP1 (3 mg/kg daily, s.c.) treatment beginning 24 hours post-irradiation completely alleviated PBI-induced 
disruption of tight junctions and adherens junctions both at 52 and 76 hours post-irradiation. These data demonstrate that $\mathrm{LPA}_{2}$ receptor activation via RP1 administered 24 hours post-irradiation can alleviate PBIinduced damage to the intestinal epithelial junctions. Conventionally, PBI-BM5 is characterized by higher dose ( $>5 \mathrm{~Gy}$ ) radiation exposure and sparing of $2.5-5.0 \%$ of bone marrow; this is considered as the ideal model for GI-ARS. TBI, characterized by ablation of $100 \%$ of bone marrow, is considered as hematopoietic acute radiation syndrome (H-ARS). Interestingly, in the present study, we show that PBI-BM5 does not affect colonic epithelial tight junction for at least 28 hours post-irradiation; tight junction disruption was observed at 52 hours and sustained at least until 100 hours post-irradiation. This is distinctly different from the effect of TBI. TBI caused tight junction disruption in less than 2 hours post-irradiation. This observation suggests that the $5 \%$ bone marrow protected by shielding during irradiation and the bone marrow-derived factors may have delayed the damage to colonic tight junction and barrier function. A new avenue of studies is necessary to understand the protective role of bone marrow in the intestinal epithelium.

Dramatic reduction of the levels of reduced-protein thiols accompanied by elevation of oxidized-protein thiols in the colon indicate that PBI-BM5 induces oxidative stress in colonic mucosa. Here we demonstrated for the first time that RP1-treatment significantly attenuates PBI-BM5-induced oxidative stress. PBI-BM5induced oxidative stress was associated with modulation of Nrf2 and antioxidant gene expression. NRF2 is a transcription factor critical for the expression of antioxidant genes (Cameron, Sekhar, Ofori \& Freeman, 2018; Jaiswal, 2004). Immunofluorescence imaging, immunoblot analysis, and Nrf2mRNA measurement by RT-qPCR indicated that PBI-BM5 caused a significant reduction of Nrf2 expression, which was prevented by RP1 at 24 hours post-irradiation. Considerable decreases in the levels of mRNA for Gpx1 , SOD1, $\operatorname{Prdx1}$, Trx 1, and CAT by PBI-BM5 and prevention of these effects by RP1indicate that in the PBI-BM5 model of irradiation the antioxidant gene expression is suppressed and that RP1 alleviates this effect of PBI. Therefore, induction of oxidative stress is likely one of the mechanisms involved in PBI-BM5-induced intestinal barrier dysfunction. Furthermore, it underlines the antioxidant action of RP1-mediated activation of $\mathrm{LPA}_{2}$ receptors in the mechanism of its protective effects.

To determine whether disruption of the actin cytoskeleton and loss of its interaction with tight junction and adherens junction are involved in the mechanism of PBI-BM5-induced disruption of the apical junctional complex, we measured the levels of detergent-insoluble fractions of tight junction and adherens junction. The detergent-insoluble fraction predominantly consists of the actin cytoskeleton. In the intact epithelium and non-disrupted tight junctions, the junctional proteins are bound to the actin cytoskeleton; therefore, they are recovered in the actin-rich detergent-insoluble fractions (Seth, Sheth, Elias \& Rao, 2007). Disruption of the actin cytoskeleton leads to disruption of tight junctions and loss of detergent-insoluble fractions of tight junction proteins (Madara, Moore \& Carlson, 1987). The present study shows that the detergentinsoluble fractions of claudin-3, E-cadherin, and $\beta$-catenin were significantly reduced by PBI-BM5, which was blocked by RP1-treatment. Data indicate that $\gamma$-irradiation disrupts the association between the actin cytoskeleton and the junctional proteins. Furthermore, it suggests that preservation of the integrity of the actin cytoskeleton may be involved in the mechanism of RP1-mediated protection of AJC in irradiated mice. The results of this study also show that $\gamma$-irradiation decreases the levels of $\mathrm{F}$-actin, likely through activating cofilin in the colonic mucosa, and RP1 treatment blocks this effect via $\mathrm{LPA}_{2}$ receptor-mediated activation of the Rho-Rock-LIM kinase pathway.

In summary, this study demonstrates that $\gamma$-irradiation disrupts AJC in the intestinal epithelium, induces mucosal barrier dysfunction, and causes endotoxemia, likely by inducing oxidative stress and disrupting the actin cytoskeleton. Furthermore, our data demonstrate that activation of the $\mathrm{LPA}_{2}$ receptor prevents and mitigates radiation-induced intestinal barrier dysfunction and endotoxemia. Therefore, $\mathrm{LPA}_{2}$ agonists like $\mathrm{RP} 1$ via the protection of the AJC of the colonic mucosa could have therapeutic benefits in the treatment of diseases associated with the disruption of the intestinal barrier.

\section{Acknowledgements}

This study was supported by the National Institute of Health grants DK55532, AA12307, CA013621, and 1UO-1AI107331. Authors thank Dr. Nathan G. Tipton (University of Tennessee Health Science Center) for 
his expert editing of the manuscript for language and scientific writing.

\section{Conflict of interest}

GT is a founder and stockholder in RxBio Inc. that has licensed the intellectual property for RP-1.

Availability of data

The data that support the findings of this study are available from the corresponding author upon reasonable request. Some data may not be made available because of privacy or ethical restrictions.

\section{References}

Anderson JM, \& Van Itallie CM (2009). Physiology and function of the tight junction. Cold Spring Harb Perspect Biol 1: a002584.

Basuroy S, Seth A, Elias B, Naren AP, \& Rao R (2006). MAPK interacts with occludin and mediates EGF-induced prevention of tight junction disruption by hydrogen peroxide. Biochem J 393: 69-77.

Basuroy S, Sheth P, Kuppuswamy D, Balasubramanian S, Ray RM, \& Rao RK (2003). Expression of kinaseinactive c-Src delays oxidative stress-induced disassembly and accelerates calcium-mediated reassembly of tight junctions in the Caco-2 cell monolayer. J Biol Chem 278: 11916-11924.

Bens M, Bogdanova A, Cluzeaud F, Miquerol L, Kerneis S, Kraehenbuhl JP, et al. (1996). Transimmortalized intestinal cells $(\mathrm{m}-\mathrm{ICcl} 2)$ that

maintain a crypt phenotype. Am J Physiol Cell Physiol 270:C1666-C1674.

Cameron BD, Sekhar KR, Ofori M, \& Freeman ML (2018). The Role of Nrf2 in response to Normal Tissue Radiation Injury. Radiat Res 190:99-106.

Chen X, \& Macara IG (2006). Par-3 mediates the inhibition of LIM kinase 2 to regulate cofilin phosphorylation and tight junction assembly. J Cell Biol 172: 671-678.

Deng W, Balazs L, Wang DA, Van Middlesworth L, Tigyi G, \& Johnson LR (2002). Lysophosphatidic acid protects and rescues intestinal epithelial cells from radiation- and chemotherapy-induced apoptosis. Gastroenterology 123: 206-216.

Deng W, Kimura Y, Gududuru V, Wu W, Balogh A, Szabo E, et al.(2015). Mitigation of the Hematopoietic and Gastrointestinal Acute Radiation Syndrome by Octadecylthiophosphate a Small Molecule Mimic of Lysophosphatidic Acid. Radiation Res 183: 465-475.

Deng W, Shuyu E, Tsukahara R, Valentine WJ, Durgam G, Gududuru V, et al. (2007). The lysophosphatidic acid type 2 receptor is required for protection against radiation-induced intestinal injury. Gastroenterology 132: $1834-1851$.

Elias BC, Suzuki T, Seth A, Giorgianni F, Kale G, Shen L, et al.(2009). Phosphorylation of Tyr-398 and Tyr-402 in occludin prevents its interaction with ZO-1 and destabilizes its assembly at the tight junctions. J Biol Chem 284: 1559-1569.

Jaiswal AK (2004). Nrf2 signaling in coordinated activation of antioxidant gene expression. Free Radic Biol Med 36: 1199-1207.

Khurana S, Tomar A, George SP, Wang Y, Siddiqui MR, Guo H, et al.(2008). Autotaxin and lysophosphatidic acid stimulate intestinal cell motility by redistribution of the actin modifying protein villin to the developing lamellipodia. Exp Cell Res 314: 530-542.

Kiss GN, Lee SC, Fells JI, Liu J, Valentine WJ, Fujiwara Y, et al. (2013). Mitigation of radiation injury by selective stimulation of the LPA(2) receptor. Biochim Biophys Acta 1831: 117-125. 
Konno T, Kotani T, Setiawan J, Nishigaito Y, Sawada N, Imada S, et al. (2019). Role of lysophosphatidic acid in proliferation and differentiation of intestinal epithelial cells. PLoS One 14:e0215255.

Kuo B, Szabo E, Lee SC, Balogh A, Norman D, Inoue A, et al.(2018). The LPA2 receptor agonist Radioprotectin-1 spares Lgr5-positive intestinal stem cells from radiation injury in murine enteroids. Cell Signal 51: 23-33.

Li C, Dandridge KS, Di A, Marrs KL, Harris EL, Roy K, et al.(2005). Lysophosphatidic acid inhibits cholera toxin-induced secretory diarrhea through CFTR-dependent protein interactions. J Exp Med 202: 975-986.

Lin FT, Lai YJ, Makarova N, Tigyi G, \& Lin WC (2007). The lysophosphatidic acid 2 receptor mediates down-regulation of Siva-1 to promote cell survival. J Biol Chem 282: 37759-37769.

Lin S, Yeruva S, He P, Singh AK, Zhang H, Chen M, et al. (2010). Lysophosphatidic acid stimulates the intestinal brush border $\mathrm{Na}(+) / \mathrm{H}(+)$ exchanger 3 and fluid absorption via LPA(5) and NHERF2. Gastroenterology 138: 649-658.

Madara JL, Moore R, \& Carlson S (1987). Alteration of intestinal tight junction structure and permeability by cytoskeletal contraction. Am J Physiol 253: C854-861.

Madara JL, Stafford J, Barenberg D, \& Carlson S (1988). Functional coupling of tight junctions and microfilaments in T84 monolayers. Am J Physiol 254: G416-423.

Pandey D, Goyal P, \& Siess W (2007). Lysophosphatidic acid stimulation of platelets rapidly induces Ca2+dependent dephosphorylation of cofilin that is independent of dense granule secretion and aggregation. Blood Cells Mol Dis 38: 269-279.

Patil R, Fells JI, Szabo E, Lim KG, Norman DD, Balogh A, et al.(2014). Design and synthesis of sulfamoyl benzoic acid analogues with subnanomolar agonist activity specific to the LPA2 receptor. J Med Chem 57: 7136-7140.

Patil R, Szabo E, Fells JI, Balogh A, Lim KG, Fujiwara Y, et al.(2015). Combined mitigation of the gastrointestinal and hematopoietic acute radiation syndromes by an LPA2 receptor-specific nonlipid agonist. Chem Biol 22: 206-216.

Rao R (2008). Oxidative stress-induced disruption of epithelial and endothelial tight junctions. Front Biosci 13: 7210-7226.

Rao RK, Basuroy S, Rao VU, Karnaky Jr KJ, \& Gupta A (2002). Tyrosine phosphorylation and dissociation of occludin-ZO-1 and E-cadherin-beta-catenin complexes from the cytoskeleton by oxidative stress. Biochem J 368: 471-481.

Rao RK, Basuroy S, Rao VU, Karnaky KJ, Jr., \& Gupta A (2002). Tyrosine phosphorylation and dissociation of occludin-ZO-1 and E-cadherin-beta-catenin complexes from the cytoskeleton by oxidative stress. Biochem J 368: 471-481.

Rao RK, Seth A, \& Sheth P (2004). Recent Advances in Alcoholic Liver Disease I. Role of intestinal permeability and endotoxemia in alcoholic liver disease. Am J Physiol Gastrointest Liver Physiol 286:G881884.

Seth A, Sheth P, Elias BC, \& Rao R (2007). Protein phosphatases 2A and 1 interact with occludin and negatively regulate the assembly of tight junctions in the CACO-2 cell monolayer. J Biol Chem 282:1148711498.

Sheth P, Samak G, Shull JA, Seth A, \& Rao R (2009a). Protein phosphatase 2A plays a role in hydrogen peroxide-induced disruption of tight junctions in Caco-2 cell monolayers. Biochem J 421:59-70.

Sheth P, Samak G, Shull JA, Seth A, \& Rao R (2009b). Protein phosphatase 2A plays a role in hydrogen peroxide-induced disruption of tight junctions in Caco-2 cell monolayers. The Biochemical journal 421: 
59-70.

Sheth P, Seth A, Atkinson KJ, Gheyi T, Kale G, Giorgianni F, et al. (2007). Acetaldehyde dissociates the PTP1B-E-cadherin-beta-catenin complex in Caco-2 cell monolayers by a phosphorylation-dependent mechanism. Biochem J 402: 291-300.

Shukla PK, Gangwar R, Manda B, Meena AS, Yadav N, Szabo E, et al.(2016). Rapid disruption of intestinal epithelial tight junction and barrier dysfunction by ionizing radiation in mouse colon in vivo: protection by N-acetyl-l-cysteine. Am J Physiol Gastrointest Liver Physiol 310: G705-715.

Shukla PK, Meena AS, Manda B, Gomes-Solecki M, Dietrich P, Dragatsis I, et al. (2018). Lactobacillus plantarum prevents and mitigates alcohol-induced disruption of colonic epithelial tight junctions, endotoxemia, and liver damage by an EGF receptor-dependent mechanism. Faseb J: fj201800351R.

Singla A, Dwivedi A, Saksena S, Gill RK, Alrefai WA, Ramaswamy K, et al. (2010). Mechanisms of lysophosphatidic acid (LPA) mediated stimulation of intestinal apical Cl-/OH- exchange. Am J Physiol Gastrointest Liver Physiol 298: G182-189.

Thompson KE, Ray RM, Alli S, Ge W, Boler A, Shannon McCool W, et al. (2018). Prevention and treatment of secretory diarrhea by the lysophosphatidic acid analog Rx100. Exp Biol Med (Maywood) 243:1056-1065.

Van Itallie CM, \& Anderson JM (2006). Claudins and epithelial paracellular transport. Annu Rev Physiol 68: 403-429.

Vardouli L, Moustakas A, \& Stournaras C (2005). LIM-kinase 2 and cofilin phosphorylation mediate actin cytoskeleton reorganization induced by transforming growth factor-beta. The Journal of biological chemistry 280: $11448-11457$.

Wang W, Halasz E, \& Townes-Anderson E (2019). Actin Dynamics, Regulated by RhoA-LIMK-Cofilin Signaling, Mediates Rod Photoreceptor Axonal Retraction After Retinal Injury. Invest Ophthalmol Vis Sci $60: 2274-2285$.

Yoshida M, He P, \& Yun CC (2016). Transgenic Expression of Human Lysophosphatidic Acid Receptor LPA2 in Mouse Intestinal Epithelial Cells Induces Intestinal Dysplasia. PLoS One 11: e0154527.

Yun CC, \& Kumar A (2015). Diverse roles of LPA signaling in the intestinal epithelium. Exp Cell Res 333: 201-207.

\section{Figure Legend}

Figure 1: LPA attenuates radiation-induced disruption of tight junctions in the intestinal epithelial monolayers .

Caco-2 and $\mathrm{m}-\mathrm{IC}_{\mathrm{C} 12}$ cell monolayers on Transwell inserts were irradiated (2-15 Gy) with or without LPA $(10 \mu \mathrm{M})$ administered $30 \mathrm{~min}$ prior to irradiation. One hour after irradiation, the tight junction integrity was examined. A \& B : Caco-2 cell monolayers exposed to radiation with or without LPA were fixed and stained for occludin (green) and ZO-1 (red). Confocal images (A) and fluorescence density at the junctions (B) are presented. Values are Mean \pm SEM $(n=4)$. The corresponding p-values (on top of the bars) indicate the significant differences between groups. NS indicates that the p-value is greater than 0.05 . C : Caco-2 cell monolayers exposed to radiation $(10 \mathrm{~Gy}$ ) with or without LPA were stained for F-actin (green) and cofilin $^{\mathrm{pS} 3}$ (red). D : Caco-2 cell monolayers were pretreated with toxin-B (TB) 30 min prior to LPA treatment. Monolayers were irradiated (10 Gy) $30 \mathrm{~min}$ after LPA treatment and stained for occludin (green), ZO-1 (red), and nucleus (blue).E : Confocal images for ZO-1 in m- $\mathrm{IC}_{\mathrm{C} 12}$ cell monolayers that were treated with LPA or vehicle prior to irradiation.

Figure 2: Effect of LPA2 receptor deficiency on the radiation-induced disruption of tight junction and adherens junction . 
Wild type (WT) and LPA2 receptor knockout (Lpar22/- ) mice were subjected to TBI (9.5 Gy). Two hours after irradiation, colonic sections were stained for tight junction and adherens junction proteins. A : Merged fluorescence images for occludin (green), ZO-1 (red), and nucleus (blue). B : Fluorescence images for ECadherin (green), $\beta$-Catenin (red), and nucleus (blue). Representative images from 4 mice per each irradiated group of mice and 2 mice for control groups are presented.

Figure 3: RP1 blocks TBI-induced disruption of tight junction and adherens junction, barrier dysfunction, and endotoxemia .

Wild type mice were injected with RP1 $(0.5 \mathrm{mg} / \mathrm{kg}$ ) or vehicle (Veh) $30 \mathrm{~min}$ prior to TBI (9.5 Gy) (IR and IR+RP1); the control group was sham-treated. At 2 hours post-irradiation, colonic sections were costained for occludin, ZO-1, and nucleus (A) or E-cadherin, $\beta$-catenin, and nucleus (B). Confocal images were captured, and fluorescence densities at the junctions were measured. Fluorescence densities for ZO-1 (C) and E-cadherin (D) are presented. At 2 and 4 hours post-irradiation, mucosal permeability in vivo in the colon (E) and ileum (F) and the plasma LPS levels $(\mathrm{G})$ were measured. Values are Mean \pm SEM $(\mathrm{n}=$ 4). The corresponding p-values (on top of the bars) indicate the significant differences between groups. NS indicates that the p-value is greater than 0.05. Experiment was repeated with similar results.

Figure 4: RP1 mitigates TBI-induced disruption of AJC, mucosal barrier dysfunction, and endotoxemia .

Adult wild type mice were subjected to TBI (9.5 Gy). At 24 hours after irradiation, mice were injected with RP1 (0.5 mg/kg daily) or vehicle (Veh); the control group was sham-treated. At 24 and 48 hours after $\mathrm{RP} 1$ treatment, junctional integrity $(\mathrm{A}-\mathrm{F})$, gut permeability in vivo $(\mathrm{G} \& \mathrm{H})$, and endotoxemia (I) were evaluated. Sections of the colon were co-stained for occludin, ZO-1, and nucleus (A), E-cadherin, $\beta$-catenin, and nucleus (B) or claudin-3, Claudin-2, and nucleus (C). Confocal fluorescence images were captured, and fluorescence densities at the junctions were measured. Fluorescence densities for ZO-1 (D) and $\beta$-catenin (E) and Claudin-3 (F) are presented. Mucosal permeabilityin vivo in the colon $(\mathrm{G})$ and ileum $(\mathrm{H})$ and the plasma LPS levels (I) were measured. Values are Mean \pm SEM $(n=4)$. The corresponding p-values (on top of the bars) indicate the significant differences between groups. NS indicates that the p-value is greater than 0.05. Experiment was repeated with similar results. Similar results were also produced in a similar experiment analyzed at 7 days post-irradiation.

Figure 5: RP1 alleviates TBI-induced downregulation of antioxidant gene expression .

Wild type (WT) mice were subjected to TBI (9.5 Gy). At 24 hours after irradiation, mice were injected with RP1 (0.5 mg/kg daily) or vehicle (Veh); the control group was sham-treated. At 24 hours after RP1 treatment, RNA extracted from colonic mucosa was analyzed for mRNA for Gpx1 (A), SOD1 (B), CAT (C), $\operatorname{Prdx1}$ (D), andNrf2 (E) by RT-qPCR. Values are Mean \pm SEM $(\mathrm{n}=4)$. The corresponding p-values (on top of the bars) indicate the significant differences between groups. NS indicates that the p-value is greater than 0.05 .

Figure 6: RP1 blocks PBI-induced disruption of AJC, barrier dysfunction, and endotoxemia .

Adult wild type mice were injected with RP1 $(3 \mathrm{mg} / \mathrm{kg})$ or vehicle (Veh) daily starting 1 day after partial body irradiation (PBI; $15.6 \mathrm{~Gy}$ ); the control group was sham-treated. At varying times after irradiation, colonic sections were co-stained for occludin, ZO-1, and nucleus (A) or E-cadherin, $\beta$-catenin, and nucleus (B). Confocal fluorescence images were captured, and the fluorescence densities of ZO-1 (C) and E-cadherin (D) at the junctions were measured. Values are Mean \pm SEM $(n=4)$. The corresponding p-values (on top of the bars) indicate the significant differences between groups. NS indicates that the p-value is greater than 0.05. Similar results were produced in similar experiment when examined at 72 and 96 hours post-irradiation.

Figure 7: RP1 mitigates PBI-induced oxidative stress .

Adult mice were injected with RP1 (3 mg/kg) or vehicle (Veh) daily starting one day after partial body irradiation (PBI-BM5; $15.6 \mathrm{~Gy}$ ); the control group was sham-treated. At 48 hours after irradiation (or 24 hours after RP1), colonic sections were stained for reduced-protein thiols, oxidized-protein thiols, and NRF2. 
Antioxidant gene expression was analyzed by RT-qPCR. A \& B : Confocal images for protein thiols were captured (A), and the fluorescence densities were measured (B). C \& D : Colonic sections were co-stained for F-actin (green), NRF2 (red) and nucleus (blue) (C). The protein extracts were immunoblotted for NRF2, and the band densities were measured.E-J : RNA isolated from colonic mucosa was analyzed for mRNA forNrf2 (E), SOD1 (F), Gpx1 (G), CAT (H), Trx1 (I), and Prdx1 (J) by RT-qPCR. Values are Mean \pm SEM $(\mathrm{n}=4)$. The corresponding $\mathrm{p}$-values (on top of the bars) indicate the significant differences between groups. NS indicates that the p-value is greater than 0.05. Similar results were produced in similar experiment when examined at 72 and 96 hours post-irradiation.

Figure 8: RP1 mitigates PBI-induced F-actin remodeling and its association with apical junctional proteins

Adult mice were injected with RP1 $(3 \mathrm{mg} / \mathrm{kg}$ ) or vehicle (Veh) daily starting one day after partial body irradiation (PBI-BM5; 15.6 Gy); the control group was sham-treated. A-E: At 48 hours after irradiation (or 24 hours after RP1), detergent-insoluble fractions of colonic mucosa were immunoblotted for tight junction and adherens junction proteins as well as NRF2 and $\beta$-actin (A). Band densities for E-cadherin (B), $\beta$ catenin (C), Cldn-3 (D), ZO-1 (E), and $\beta$-actin (F) were measured. G: Cryosections of the colon were fixed and stained for F-actin (red) and nucleus (blue). H \& I: Colonic sections were co-stained for cofilin ${ }^{\mathrm{pS} 3}$ (red), F-actin (green) and nucleus (blue) $(\mathrm{H})$. Mucosal extracts were immunoblotted for cofilin ${ }^{\mathrm{pS} 3}$ and $\beta$ actin (I). Band densities were measured, and the cofilin ${ }^{\mathrm{pS} 3}$ band densities presented by values normalized to corresponding actin band densities (I). In all graphs, values are Mean \pm SEM $(n=4)$. The corresponding p-values (on top of the bars) indicate the significant differences between groups. NS indicates that the p-value is greater than 0.05 . 

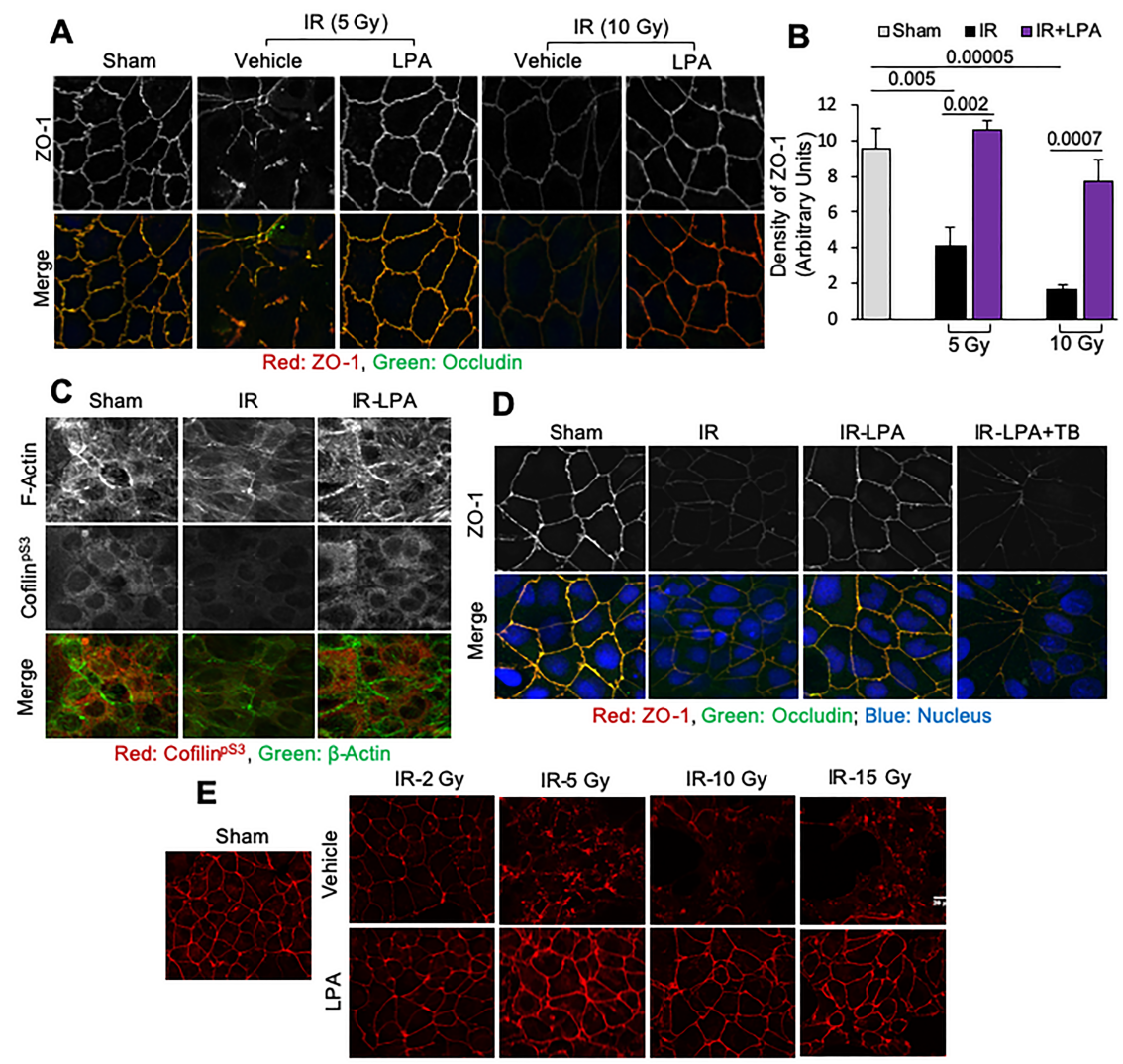

FIG. 1 


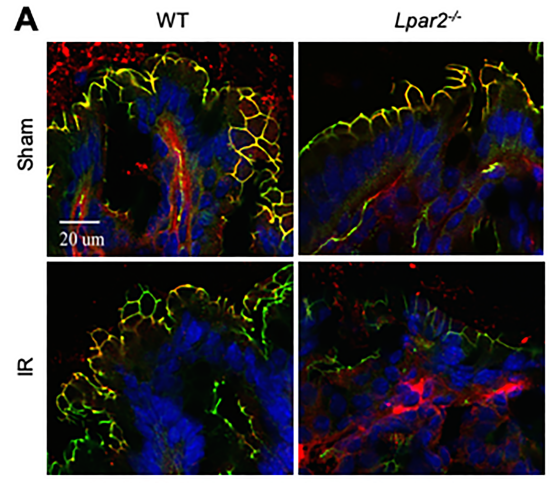

Red: ZO-1, Green: Occludin; Blue: Nucleus

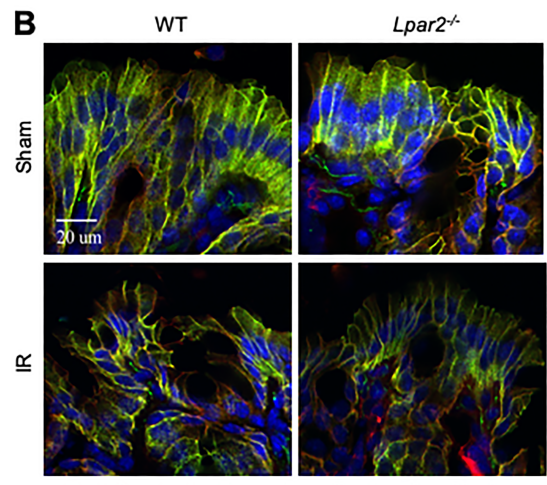

Red: $\beta$-catenin, Green: E-cadherin; Blue: Nucleus

FIG. 2 

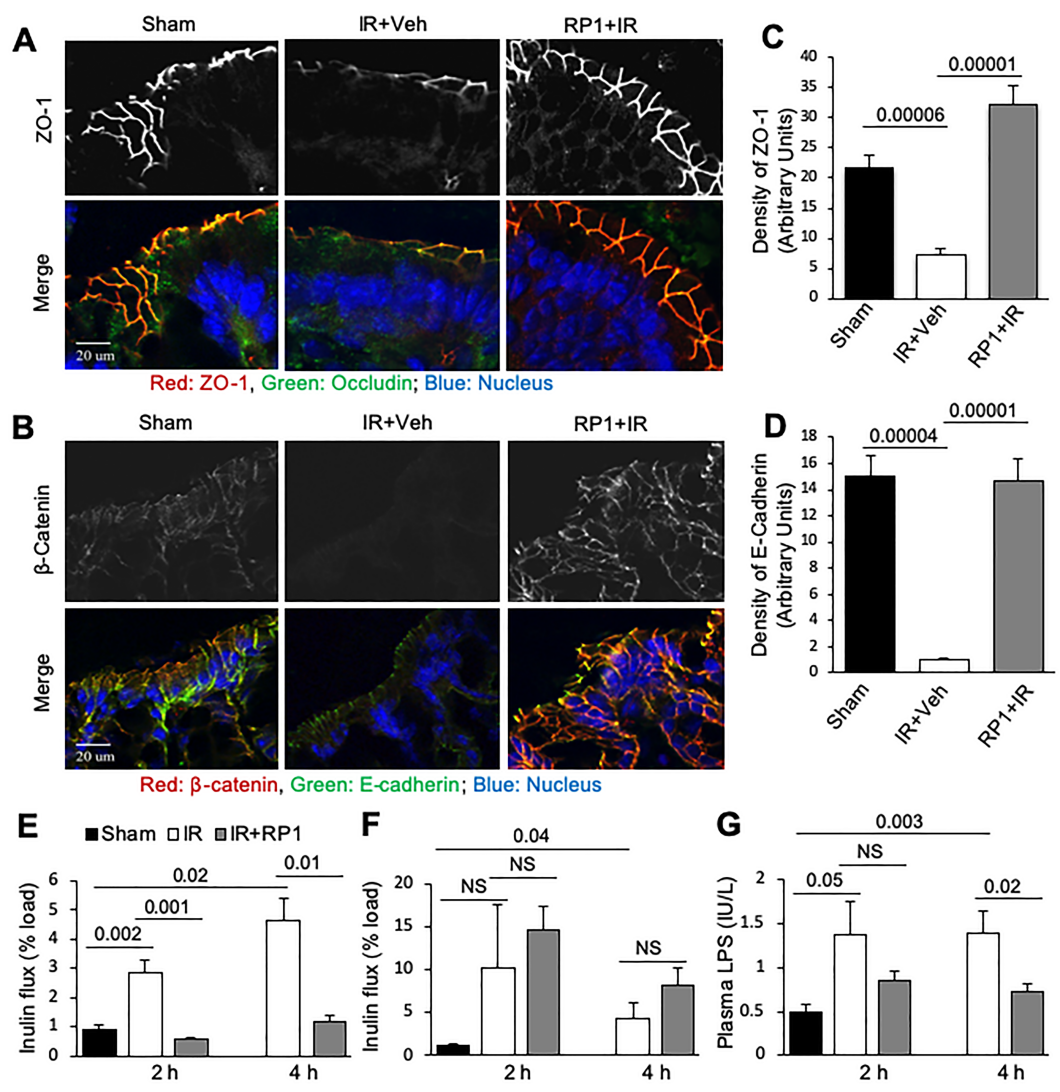

FIG. 3 

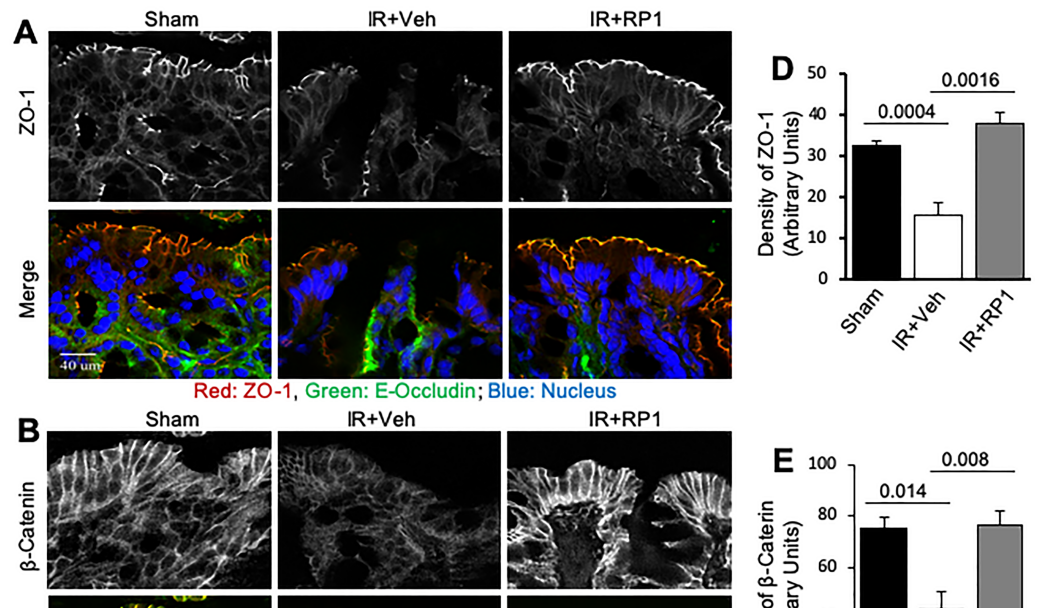

IR+RP1

휸


$\mathrm{IR}+\mathrm{Veh}$

IR+RP1


Red: Claudin-3, Green: Claudin-2; Blue: Nucleus
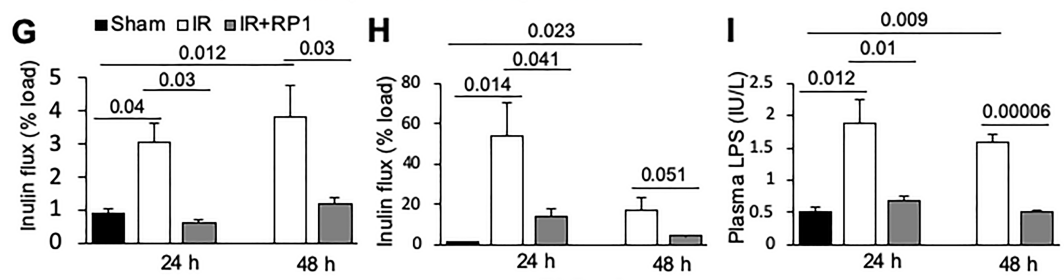

FIG. 4 




FIG. 5 

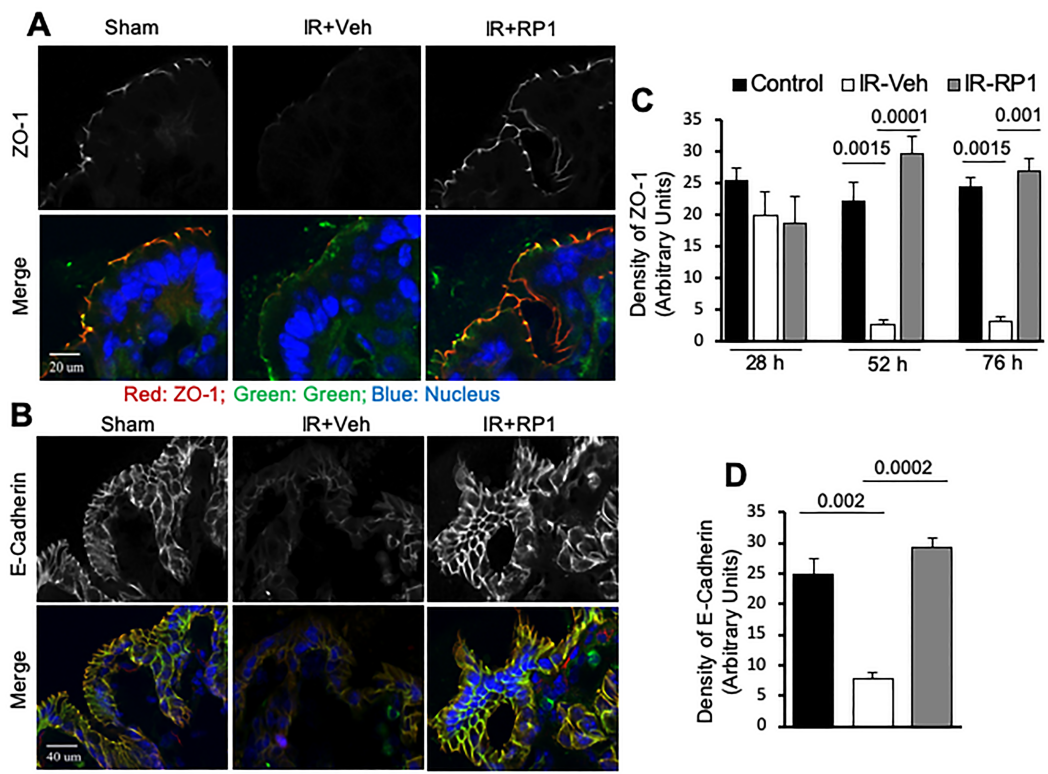

Red: $\beta$-Catenin; Green: E-Cadherin; Blue: Nucleus

FIG. 6 


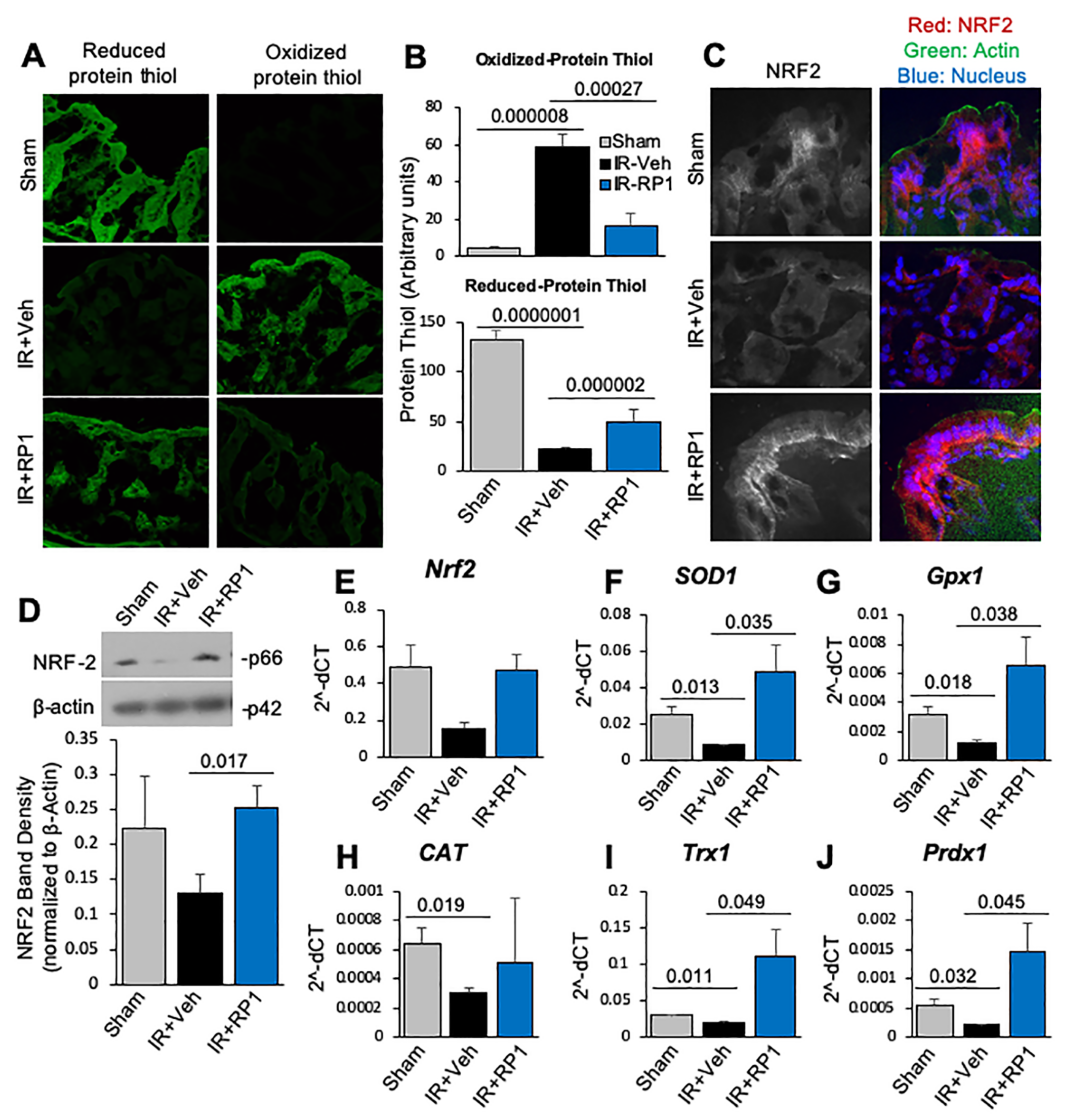

FIG. 7 



Red: Cofilin ${ }^{p S 3}$; Green: Actin; Blue: Nucleus

FIG. 8 